\title{
A review of potential pharmacological treatments of COVID-19: an evidence-based perspective
}

\author{
Vikas Kumar, Gyan Vardhan, Kalpana Tiwari, Puneet Dhamija*
}

${ }^{1}$ Department of Pharmacology, All India Institute of Medical Sciences, Rishikesh, Uttarakhand, India

Received: 19 August 2020

Revised: 23 September 2020

Accepted: 28 September 2020

\section{*Correspondence:}

Dr. Puneet Dhamija,

Email: puneet.phar@aiimsrishikesh.edu.in

Copyright: () the author(s), publisher and licensee Medip Academy. This is an open-access article distributed under the terms of the Creative Commons Attribution Non-Commercial License, which permits unrestricted non-commercial use, distribution, and reproduction in any medium, provided the original work is properly cited.

\begin{abstract}
Coronaviruses (CoVs) typically manifest as mild to severe respiratory tract infections. No drug is approved by US food and drug administration (FDA) for the treatment of patients with coronaviruses infection. With growing COVID19 pandemic globally, need of hour is to work on potential prophylactic and therapeutic drugs to prevent local and community transmission. A literature search for eligible studies published till March 2020 was conducted in the PubMed, Medline, EMBASE, OVID, and Google Scholar databases by two reviewers. Therapeutic efficacy and safety of different drug regimens targeting treatment pathway acting against corona virus-2019 (COVID-19) were reviewed. Possible mechanism of actions of these potential repurposed drugs against COVID-19 were reviewed to develop effective prevention and treatment strategies. Many potential pharmacological therapies are being studied in various clinical trials. No FDA-approved repurposed drugs have shown safety and efficacy in randomized controlled trials for patients with COVID-19. Vaccines are under development and only few vaccines are under clinical evaluation. This review highlights potential drug actions against COVID-19 and their safety issues. It could help researchers and physicians to use these potential agents judiciously in clinical trials as well as in treatment protocols.
\end{abstract}

Keywords: Coronavirus infection, SARS, COVID-19, Pharmacotherapy, Potential targets, Repurposed drugs

\section{INTRODUCTION}

Coronavirus $(\mathrm{CoV})$ typically manifest as mild to severe respiratory tract infections. ${ }^{1}$ It resulted in global pandemic in the past couple of decades with two highly pathogenic human strains (HCoVs): severe acute respiratory syndrome coronavirus (SARS-CoV) and Middle East respiratory syndrome coronavirus (MERS$\mathrm{CoV})$ leading to high morbidity and mortality. ${ }^{2}$ Phylogenetically, 2019-novel CoV/SARS-CoV-2 resembles nucleotide sequence identity with SARS-CoV (79.7\%). The evolutionarily conserved regions, envelope and nucleocapsid proteins of 2019-nCoV/SARS-CoV-2, have sequence identities of $96 \%$ and $89.6 \%$ respectively with SARS-CoV. ${ }^{3}$
World health organization (WHO) estimated MERS-CoV caused a total of 2494 diagnosed cases and 858 deaths (majority in Saudi Arabia). ${ }^{2}$ SARS-CoV-2 outbreak in Wuhan, China caused over 8,73,541 cases and 43,294 deaths. ${ }^{4}$ The basic reproductive number (R0) was reported to be approximately 2-3, signifies on average each infected patient has the potential to spread infection to 2-3 other individuals. As Ro>1, it was assumed that the epidemic shall continue spreading in days to come. ${ }^{5}$ Children and elderly were affected more during outbreak. It was observed that $27 \%$ of patients sought medical help within 2 days of onset however $89 \%$ of patients were not hospitalized until day 5 of illness. ${ }^{4}$ The interval between the onset of illness and seeking medical attention was short but there were delays in hospitalization. The data on incubation period suggests an observation period or 
quarantine for 14 days to exposed individuals. Allocating resources for rapid testing is therefore essential to ensure early testing, contain patients and limit the disease spread. It is also essential to diagnose asymptomatic cases and to ascertain severity of infection. ${ }^{6}$

Presently, there are no effective medications or vaccine available for this disease. Clinical management of COVID-19 infection includes, prevention and control measures and supportive care, including supplementary oxygen and mechanical ventilatory support when indicated. ${ }^{6}$ Many repurposed as well as several investigational drugs are being studied in various clinical trials that are underway across the globe. Quarantine, isolation, supportive care for those who become ill and infection-control measures are mainstay strategies to prevent disease spread and control infection. There is lack of specific antiviral agent to treat the infected and optimally decrease viral shedding and subsequent transmission. $^{7}$ With growing COVID-19 pandemic globally, need of hour is to work on prophylactic drugs to prevent local and community transmission.

\section{METHODS}

We performed a review of studies investigating therapeutic efficacy and safety of different drug regimens targeting treatment pathway acting against coronavirus infection (COVID-19). We also assessed possible mechanism of this combination as repurposed drug against COVID-19 for development of effective prevention and treatment strategies.

\section{Search strategy}

A literature search for eligible studies published till July 2020 was conducted in the PubMed, Medline, EMBASE, and Google Scholar databases by two reviewers. The following combinations of main keywords were used: (Novel Corona Virus) and (COVID-19), (2019nCoV/SARS-CoV-2), (Chloroquine/Hydroxychloro quine) and (Ivermectin), (Azithromycin), (Lopinavir and ritonavir), (Oseltamivir), (Remdesivir), (Favipiravir), (Tocilizumab), (Sarilumab), (Baricitinib), (Itolizumab), (vaccine), (convalescent plasma), (Viral infection management), (Potential Pharmacotherapy). Active clinical trials were identified using the disease search term coronavirus infection on clinicaltrials.gov. All eligible studies were screened by two more reviewers following published guidelines.

\section{OBSERVATIONS}

\section{Antiparasitics}

\section{Chloroquine/Hydroxychloroquine}

The hypothesis of using chloroquine/ hydroxychloroquine (HCQ) as prophylaxis is, its ability to supress cytokine release syndrome because of immune system overactivation by SARS-CoV-2 infection. HCQ inhibit lysosomal activity in antigen-presenting cells (APCs), plasmacytoid dendritic cells (pDCs) and B cells, by raising intracellular $\mathrm{pH}$. It prevents antigen processing and major histocompatibility complex (MHC) class IImediated autoantigen presentation to $\mathrm{T}$ cells. ${ }^{8}$ It further inhibits $\mathrm{T}$ cell activation and expression of co-stimulatory proteins and cytokines. ${ }^{9,10}$ It also supresses toll-like receptor (TLR) signalling due to the altered $\mathrm{pH}$ of endosomes and interrupts binding between TLR and their RNA/DNA ligands. ${ }^{11}$ HCQ also interferes with the interaction between cytosolic DNA and the nucleic acid sensor cyclic GMP-AMP (cGAMP) synthase (cGAS), in the cytoplasm. ${ }^{12}$ Pro-inflammatory signalling activation and cytokines production get inhibited as both TLR signalling and cGAS stimulation of interferon genes are suppressed. ${ }^{10}$ According to Indian council of medical research (ICMR), HCQ is indicated for asymptomatic healthcare workers involved in the care of suspected or confirmed cases of COVID-19: $400 \mathrm{mg}$ twice a day on Day 1 , followed by $400 \mathrm{mg}$ once weekly for next 7 weeks; and asymptomatic household contacts of laboratory confirmed cases: $400 \mathrm{mg}$ twice a day on Day 1, followed by $400 \mathrm{mg}$ once weekly for next 3 weeks; to be taken with meals. The safety concerns related to HCQ are, risk of cardiac arrhythmias (e.g. QT prolongation), risk of retinal damage, especially with long term use, caution in patients with G6PD deficiency, caution in diabetics, significant drug interactions.

\section{Ivermectin}

Ivermectin is FDA-approved anti-parasitic agent. It has shown broad-spectrum anti-viral activity in vitro. Recently, it was found to inhibit SARS-CoV-2, with a single addition to Vero-hSLAM cells with $5 \mu \mathrm{M}$ ivermectin, 2 hours post infection with SARS-CoV-2. It was effective in reducing $~ 5000$-fold in viral RNA at 48 hours. ${ }^{13}$ The IC50 of ivermectin treatment was determined to be $\sim 2 \mu \mathrm{M}$. The common safety issues include, Headache, dizziness, muscle pain, nausea, or diarrhoea.

\section{Antibiotics}

\section{Azithromycin}

Azithromycin is a broad-spectrum macrolide antibiotic with a long half-life and a high degree of tissue penetration. It has additional immunomodulatory effects and used in respiratory inflammatory diseases. Antiinflammatory effects include decrease in proinflammatory cytokine production and increase in macrophages phagocytosis ability. Direct antiviral action is also reported, but mechanism remains unclear. ${ }^{14}$ Potential immunomodulatory effects of azithromycin is reported that includes, its ability to down-regulate inflammatory process, decreasing production of reactive oxygen species and airway mucus secretion, inhibiting bacterial biofilm, neutrophil activation and mobilization, accelerating neutrophil apoptosis, and blocking the activation of 
nuclear transcription factors. It interferes with ERK signal transduction and reduces mucin production as well as neutrophil migration. ${ }^{15}$ The dosing schedule implemented in a clinical study was $500 \mathrm{mg}$ on day 1 followed by 250 mg per day, the next four days in combination with HCQ $(600 \mathrm{mg}) .{ }^{16}$ Safety issue related to azithromycin and hydroxychloroquine combination is, increased risk of cardiac arrhythmia i.e., QTc prolongation in ECG. ${ }^{17}$ Other side effects of azithromycin include, gastrointestinal upset (abdominal pain, diarrhea, constipation, nausea), dizziness, headaches, photosensitivity, or skin rash.

\section{Antivirals}

\section{Lopinavir-ritonavir with ribavirin combination}

Lopinavir and ritonavir are antiretroviral protease inhibitors and ribavirin is nucleoside inhibitor. The 306 amino acids long main protease (Mpro) is a key enzyme for coronavirus replication. Studies have shown that Mpros of different coronaviruses are highly conserved in terms of both sequences and 3D structures. Lopinavir and ritonavir may bind to Mpro, a key enzyme for coronavirus replication.18 In an open-label, individually randomized, controlled trial study, dose schedule followed for lopinavir-ritonavir was $400 \mathrm{mg}$ and $100 \mathrm{mg}$, orally, twice daily for 14 days. ${ }^{19}$ The dosing schedule for ribavirin administered in a clinical study for SARS was, $2.4 \mathrm{~g}$ orally as a loading dose followed by $1.2 \mathrm{~g}$ orally every 12 hours and duration of treatment up to 10 days. ${ }^{20}$ The reported safety concerns related to Lopinavir-ritonavir include, gastrointestinal adverse events including nausea, vomiting, and diarrhoea.

\section{Oseltamivir}

Oseltamivir phosphate is a pro-drug of the active metabolite (oseltamivir carboxylate). It is a potent selective inhibitor of influenza virus neuraminidase enzymes present on the virion surface. Viral neuraminidase enzyme activity is important for viral entry into uninfected cells, for the release of recently formed virus particles from infected cells, and for the further spread of the infectious virus in the body. Oseltamivir activity reduces viral infectivity and shedding. Oseltamivir is recommended for people at high risk of infection before or after exposure to pandemic influenza. $^{21}$ The effective dose for oseltamivir is $75 \mathrm{mg}$ once daily. It is generally well tolerated. Few reported safety issues related to it include, anaphylactic reactions, Stevens-Johnson syndrome, toxic epidermal necrolysis, and erythema multiforme.

\section{Remdesivir}

Remdesivir is a 1'-cyano-substituted adenosine nucleotide analogue with broad-spectrum antiviral activity against various RNA viruses. The compound undergoes a metabolic mechanism, activating nucleoside triphosphate (NTP) metabolite for inhibiting viral RNA polymerases. It competes with adenosine-triphosphate (ATP) for nascent viral RNA chains at position $i$ and terminates RNA synthesis at position $\mathrm{i}+3$. It causes delayed chain termination and evade proofreading by viral exoribonuclease. ${ }^{22}$ The dosing schedule used in clinical trials is, $200 \mathrm{mg}$ loading dose on day 1, followed by 100 $\mathrm{mg}$ iv once-daily maintenance doses for 9 days. ${ }^{23}$ Safety issues are elevated liver enzymes and gastrointestinal symptoms.

\section{Favipiravir}

Favipiravir a purine nucleic acid analogue, selectively and potently inhibits the RNA-dependent RNA polymerase (RdRp) of RNA viruses. Favipiravir undergoes an intracellular phosphoribosylation to be an active form, favipiravir-RTP (favipiravir ribofuranosyl-5'triphosphate), which is recognized as a substrate by RdRp, and inhibits the RNA polymerase activity. It inhibits RdRp of the influenza virus with an IC50 of $0.022 \mu \mathrm{g} / \mathrm{ml}$, but does not affect the human DNA polymerases $\alpha, \beta, \gamma$ subunits at up to $100 \mu \mathrm{g} / \mathrm{ml}^{24}$ Genome sequencing of the 2019-nCoV identified the virus as a single-stranded RNA beta-coronavirus with the RdRp gene similar to those of SARS-CoV and MERS$\mathrm{CoV} .{ }^{25}$ Therefore, favipiravir is considered as one of the potential candidates for COVID-19. Favipiravir is usually well tolerated but has concerns regarding teratogenic risks.

\section{Sofosbuvir}

Sofosbuvir is FDA approved directly acting antiviral (DDA) drug against RNA dependent RNA polymerase (RdRp) of Hepatitis C. It was tested in-silico, as directacting antiviral (DAA) drugs against COVID-19 RdRp model. Results were found to be promising against Corona Virus inhibition. ${ }^{26}$

\section{Immunomodulators}

\section{Tocilizumab}

These are monoclonal antibodies against IL-6. It is postulated that cytokine release syndrome may be a component of severe COVID-19 infection. Tocilizumab inhibits IL-6-mediated signalling pathway by competitively binding to both soluble and membranebound IL-6 receptors. IL-6 is a proinflammatory cytokine that is involved in diverse physiological processes such as T-cell activation, immunoglobulin secretion induction, hepatic acute-phase protein synthesis initiation, and hematopoietic precursor cell proliferation and differentiation stimulation. The dose administered in a study was $400 \mathrm{mg}$ once through an intravenous drip. ${ }^{27}$ The safety issues related to it include, risk of GI perforation, risk of hepatotoxicity, caution in patients with thrombocytopenia and neutropenia and infusion-related reactions. 


\section{Sarilumab}

Sarilumab inhibits IL-6 that might play a role in driving the inflammatory immune response that causes acute respiratory distress syndrome observed in patients with severe COVID-19 infection. It is a potential drug candidate in patients with moderate, severe pneumonia or critical pneumonia associated with COVID-19. The dose administered in a phase $2 / 3$ randomized clinical trial as $400 \mathrm{mg}$, single intravenous dose for 1 hour-infusion. ${ }^{28}$ Common adverse drug reactions associated with it are, neutropenia, injection-site erythema, increased lowdensity lipoprotein (LDL) cholesterol, and increased liver enzymes.

\section{Baricitinib}

Baricitinib is a AP2-associated protein kinase 1 (AAK1) binding drug that inhibits Janus Kinase, also binds cyclin G-associated kinase, another regulator of endocytosis. ${ }^{29}$ the plasma concentration of baricitinib on therapeutic dosing (either as $2 \mathrm{mg}$ or $4 \mathrm{mg}$ once daily) is sufficient to inhibit AAK1, we suggest it could be trialled, using an appropriate patient population with 2019-nCoV acute respiratory disease, to reduce both the viral entry and the inflammation in patients.

\section{Type 1 interferons (IFN-I)}

IFN-I plays a major role in antiviral immunity because of immunomodulatory properties. Interferon treatment has shown mixed efficiency against SARS-CoV and MERSCoV. IFN $\beta 1$ is suggested to be safe and easy to upscale treatment against COVID-19 in the early stages of infection. ${ }^{30}$ In a phase 3, multi-centre, adaptive, randomized, open label, clinical trial of the safety and efficacy of treatments of COVID-19 in hospitalized adults, Interferon Beta-1A provided as single-dose prefilled graduated syringe with $44 \mu \mathrm{g}$ per $0.5 \mathrm{ml}$ is undertrial. $^{31}$ Safety issues are injection site infection, headache, flu-like syndrome, muscle ache, fever, asthenia, nausea, chills, diarrhoea, and dizziness.

\section{Itolizumab}

Itolizumab, an anti-CD6 humanized IgG1 mAb approved by the FDA and drug controller general of India (DCGI) for the treatment of moderate to severe chronic plaque psoriasis. Its (IgG1) isotype binds to domain 1 of human CD 6. It modulates T-lymphocytes activation and proliferation induced by CD6-costimulation and regulates downstream pathways such as pMAPK, pSTAT3 and $\mathrm{pAKT}$ that further results in reduction of INF- $\gamma, \mathrm{TNF} \alpha$ and IL- 6 both in vitro and in vivo. The safety and efficacy of itolizumab in COVID 19 was evaluated in a multicentric, open label, two Arm Randomized, pivotal phase 2 study with 30 participants. Starting dose was $1.6 \mathrm{mg} / \mathrm{kg}$ dose iv infusion, if well tolerated and patient showed improvement, it was either continued with $1.6 \mathrm{mg} / \mathrm{kg}$ dose every 2 weeks or $0.8 \mathrm{mg} / \mathrm{kg}$ weekly regimen. ${ }^{32}$ All patients treated with itolizumab responded positively and recovered. There were deaths in the control arm that did not involve treatment with itolizumab in a 1-month mortality rate evaluation. ${ }^{33}$ The most common adverse effect noted was infusion reactions; frequency and severity decreased with subsequent infusions and symptoms included nausea, rash, urticaria, flushing, cough, wheezing, dyspnoea, dizziness, and headache. ${ }^{34}$ In some cases, severe reactions were seen leading to a further oxygen decompensation state in COVID-19 infected patients. It was observed during the first cycle of dosing and tend to decrease in severity and frequency upon subsequent infusions. It is recommended that infusion should be administered slowly over 5-6 hours to reduce the incidence of infusion reactions and better tolerability. ${ }^{35}$

\section{Vaccines}

The development of COVID-19 vaccines is a global priority for ending the pandemic. A single-centre, open and dose-escalation phase I clinical trial for recombinant novel coronavirus vaccine (adenoviral vector) in healthy adults aged between 18 and 60 years is underway. ${ }^{36}$ Another phase I, open-label, dose-ranging study of the safety and immunogenicity of 2019-nCoV Vaccine (mRNA-1273) in 45 healthy adults is under process. ${ }^{37}$

Besides these, a placebo-controlled adaptive multi-centre randomized controlled trial on bacille Calmette Guerin (BCG) vaccine versus placebo, in healthcare workers with direct patient contacts among which nurses and physicians working at emergency rooms and wards where COVID-19-infected patients are treated is underway to reduce absenteeism among them. Dose administered is intracutaneously $0.1 \mathrm{ml} \mathrm{BCG}$ vaccine, that accounts for $0.075 \mathrm{mg}$ of attenuated Mycobacterium bovis. ${ }^{38}$

COVAXIN TM vaccine by bharat biotech is developed in collaboration with ICMR - national institute of virology (NIV). This indigenous, inactivated vaccine is developed and manufactured in Bharat Biotech's BSL-3 (Bio-Safety Level 3) high containment facility. The vaccine received DCGI approval for phase I and II human clinical trials and the trials commenced across India from July 2020. ${ }^{39}$ Phase 1 to be followed by phase 2 randomized, doubleblind, multicentre study. The study was designed to evaluate the safety, reactogenicity, tolerability, and immunogenicity of three groups of healthy volunteers who received two intramuscular doses of BBV152 vaccine formulations. A total sample size of 1125 healthy volunteers, with 375 volunteers in the phase 1 study and 750 volunteers in phase 2 study (4:1 test and control). ${ }^{40}$ Another recombinant vaccine AZD1222 from the University of oxford and AstraZeneca is under development. ${ }^{41}$ Serum Institute of India has partnered with AstraZeneca and Oxford University to supply AZD 1222 vaccine. $^{42}$ A replication-deficient chimpanzee adenovirus was used to carry DNA for the spike antigen protein of SARS-CoV-2 that enabled the virus to infect 
human cells. Due to the immunogenicity of adenoviruses, vaccine could provide better protection with fewer doses than other candidate vaccines. The Oxford team was able to pull ahead in the race for a vaccine because it had already completed safety testing on a similar vaccine candidate in 2019, for Middle East respiratory syndrome coronavirus. AZD1222 has now entered phase 2 and 3 trials in the UK. ${ }^{41}$

\section{Convalescent plasma}

The use of convalescent plasma was recommended as an empirical treatment during outbreaks of Ebola virus in 2014, and a protocol for treatment of middle East respiratory syndrome coronavirus with convalescent plasma was established in 2015. This approach with other viral infections such as SARS-CoV, H5N1 avian influenza, and $\mathrm{H} 1 \mathrm{~N} 1$ influenza also suggested that transfusion of convalescent plasma was effective. In an uncontrolled case series of five critically ill patients with COVID-19 and ARDS, administration of convalescent plasma containing neutralizing antibody led to improvement clinical status of patients. ${ }^{43}$

\section{Melatonin}

Melatonin is released from pineal gland. L-tryptophan is a precursor for the synthesis of melatonin. It binds to melatonin receptor type $1 \mathrm{~A}$, that acts on adenylate cyclase and inhibits a cAMP signal transduction pathway. Melatonin inhibits adenylate cyclase and also activates phospholipase $\mathrm{C}$, that potentiates release of arachidonate. It binds to receptors 1 (MT1) and 2 (MT2) that triggers downstream signaling cascades which results various effects in the body. The melatonin receptors are $G$ protein-coupled receptors and are expressed in various tissues of the body. Both MT1 and MT2 receptors are expressed in central nervous system (CNS), coronary circulation and aorta and the immune system. The activation of MT1 receptor, inhibits the adenylyl cyclase and causes decreased formation of cyclic adenosine monophosphate (cAMP), and then less protein kinase A (PKA) activity, which in turn hinders phosphorylation of cAMP responsive element-binding protein (CREB binding protein) into P-CREB. It also activates phospholipase $\mathrm{C}$ (PLC) and regulates influx of ion inside the cell. MT2 receptors activation, inhibits adenylyl cyclase and guanylyl cyclase which decreases the formation of cAMP and cyclic guanosine monophosphate (cGMP), respectively. Binding to MT2 receptors probably affects PLC which increases protein kinase C (PKC) activity. ${ }^{44}$

Melatonin is not likely to have an antiviral property. Therefore, the use melatonin in COVID-19 is not for the purpose to eradicating the virus or even curbing its proliferation (although it may have this effect). The strategy for melatonin application is for the purpose of retarding the body's excessive immunoinflammatory responses caused by virus invasion. ${ }^{45}$

\section{Corticosteroids}

In preliminary report of (Randomised Evaluation of COVID-19 therapy) recovery Trial, a widely used corticosteroid, low dose dexamethasone was found to be effective in seriously ill patients of COVID-19. Out of total 11500 patients enrolled, 2104 were randomised to receive dexamethasone $6 \mathrm{mg}$ once daily (orally or intravenously) for ten days and were compared with 4321 patients on standard of care, with follow-up of 28 days. Mortality was more in patients who received standard of care and required ventilation $(41 \%)$, moderate who required oxygen only $(25 \%)$, and lowest among those who were not on any respiratory intervention (13\%). Dexamethasone reduced mortality by one-third in patients on ventilator and one-fifth who required oxygen. No beneficial effect was seen in those who did not require any respiratory intervention. ${ }^{46}$ The documented adverse drug reactions related to corticosteroids are gastrointestinal intolerance, menstrual irregularities, dizziness, headache, insomnia and weight gain.

\section{Drugs not recommended or cautious use}

NSAIDs and Ibuprofen are not recommended in COVID19 cases because of potential worsening of symptoms. It is postulated that ibuprofen might increase the expression of ACE-2 that can further worsen COVID-19 positive patients because of tropism effect.

\section{Risk of COVID-19 infection with angiotensin- converting enzyme 2 and angiotensin II type-I receptor blockers}

Human pathogenic coronaviruses (severe acute respiratory syndrome coronavirus [SARS-CoV] and SARSCoV-2) bind to their target cells through angiotensin-converting enzyme 2 (ACE2), which is expressed by epithelial cells of the lung, intestine, kidney, and blood vessels. The expression of ACE2 is substantially increased in patients with type 1 or type 2 diabetes, who are treated with ACE inhibitors and angiotensin II type-I receptor blockers (ARBs). Upregulation of ACE2 occurs due to treatment with ACE inhibitors and ARBs in hypertension and diabetes mellitus. Therefore, it was suggested that patients with cardiac diseases, hypertension, or diabetes, who are treated with ACE2- increasing drugs, are at higher risk for severe COVID-19 infection. However, lack of robust evidences warrants plausibility. ${ }^{47,48}$

\section{DISCUSSION}

Currently, WHO has recommended hydroxychloroquine, corticosteroids, remdesivir and anti- retroviral drugs for treatment of COVID-19. Hydroxychloroquine has in vitro activity against SARS-CoV-2 and may have immunomodulating properties. ${ }^{49,50}$ An open-label, nonrandomized clinical trial compared hydroxychloroquine treatment $(n=26)$ to an untreated negative control group. 
On day $6,70 \%$ of hydroxychloroquine-treated patients were virologically cured compared to $12.5 \%$ in the untreated control group. ${ }^{15}$ A large-scale observational study of HCQ in hospitalized patients with COVID 19 was conducted to examine association with its use and intubation or death. It was found to be not associated with either a greatly lowered or an increased risk of the composite end point of intubation or death. ${ }^{51}$ Several clinical trials evaluating the efficacy of remdesivir in patients infected with SARS-CoV-2 are currently being conducted. $^{14}$ In preclinical trials, remdesivir has demonstrated significant activity against coronavirus and a high genetic barrier to resistance. ${ }^{22}$ A double-blind, randomized placebo controlled trial of intravenous remdesivir in 1063 adults hospitalized with COVID-19 with lower respiratory tract involvement was conducted. Remdesivir was found to be superior to placebo in shortening the time to recovery. ${ }^{52}$ In an open label, nonrandomized clinical trial, hydroxychloroquine and azithromycin treatment combination administered in twenty COVID-19 patients, significantly reduced viral load. ${ }^{16}$ In a randomized, controlled, open-label trial involving hospitalized adult patients with confirmed SARS-CoV-2 infection, no benefit was observed with lopinavir-ritonavir treatment beyond standard care. ${ }^{19}$ The results for clinical trial including lopinavir-ritonavir with ribavirin is still awaited. However, favourable clinical response and significantly lower adverse outcomes were observed with lopinavir-ritonavir with ribavirin combination in another clinical study. ${ }^{53} \mathrm{~A}$ randomized, controlled, open-label trial involving 199 adult hospitalized patients with laboratory confirmed SARSCoV2 was conducted to evaluate effect of lopinavirritonavir on clinical improvement. No benefit was observed with lopinavir-ritonavir treatment beyond standard care. ${ }^{54}$ A randomized, open, controlled small sample clinical study is underway to assess the efficacy of oseltamivir with other drug combinations for 2019-nCoV pneumonia patients. ${ }^{55}$ Sofosbuvir tightly binds to the Corona Virus RdRp in-silico model and block the function of the protein leading to viral eradication. ${ }^{26}$ However, further optimization, in-vitro and in-vivo and clinical trials need to be conducted to establish its efficacy and safety against corona virus infection.

In a study involving severe to critical cases of COVID-19, tocilizumab found to effectively improve clinical symptoms and repress the deterioration of severe COVID19 patients. ${ }^{27}$ Itolizumab is recommended for the emergency treatment of acute respiratory distress syndrome (ARDS) in patients with COVID 19. ${ }^{33}$

There are almost 70 candidate vaccines according to WHO and three among them under clinical trial. ${ }^{56}$ Convalescent plasma containing neutralizing antibody is a promising therapeutic option for clinical improvement in critically ill patients. No FDA-approved drugs have shown safety and efficacy in randomized controlled trials for patients with COVID-19. Meticulously designed randomized controlled trials should ideally be done to demonstrate efficacy and safety of investigational therapies for treatment of COVID-19. Several clinical trials are underway testing multiple drugs with in-vitro antiviral activity against SARS-CoV-2 and/or immunomodulatory effects that may have clinical benefit. A special emergency program for possible therapies is launched by U.S. FDA, the Coronavirus Treatment Acceleration Program (CTAP). This initiative would lead to movement of new treatments to patients as quickly as possible, while at the same time finding out whether they are effective and safer. ${ }^{57}$

Melatonin was reported in potential antiviral infection via its anti-inflammatory and antioxidant effects.58 Melatonin indirectly regulates ACE2 expression, a key entry receptor involved in viral infection of $\mathrm{HCoVs}$, including 2019-nCoV/SARS-CoV-2. ${ }^{59}$ Melatonin applica tion retards the body's excessive immunoinflammatory responses. ${ }^{45}$ It lowers the level of IL-6, IL-8 and TNFalpha that induces acute respiratory distress syndrome, a cardinal sign in COVID-19 severe cases. ${ }^{60}$

Dexamethasone emerged as first lifesaving drug for COVID-19 treatment in RECOVERY Trial. ${ }^{61}$ Although there are many documented safety issues related to corticosteroids, evaluating benefit to risk ratio, corticosteroids are favourable for seriously ill patients of COVID-19. Initially, in an interim guidance of WHO Corticosteroid was not recommended for treatment of COVID-19 cases with pneumonia. However, it was recommended to be used with caution in critical patients with Acute respiratory distress syndrome. ${ }^{62}$

\section{CONCLUSION}

Currently, neither any specific treatment nor vaccine available for 2019-nCoV/SARS-CoV-2. There is an urgent need for the development of effective prevention and treatment strategies for 2019-nCoV/SARS-CoV-2 outbreak. This review highlights potential drug actions against COVID-19 and their safety issues. This could definitely help researchers and physicians to use these potential agents judiciously in clinical trials as well as in treatment protocols.

\section{Funding: No funding sources Conflict of interest: None declared \\ Ethical approval: Not required}

\section{REFERENCES}

1. Zumla A, Chan JFW, Azhar EI, Hui DSC, Yuen KY. Coronaviruses- drug discovery and therapeutic options. Nat Rev Drug Discov. 2016;15(5):327-47.

2. WHO. Middle East respiratory syndrome coronavirus (MERS-CoV). Available from: https://www.who.int/ emergencies/mers-cov/en. Accessed on 20 March 2020 .

3. Chen N, Zhou M, Dong X, Qu J, Gong F, Han Y, et al. Epidemiological and clinical characteristics of 99 
cases of 2019 novel coronavirus pneumonia in Wuhan, China: a descriptive study. Lancet Lond Engl. 2020;395(10223):507-13.

4. Bauch CT, Lloyd-Smith JO, Coffee MP, Galvani AP. Dynamically modeling SARS and other newly emerging respiratory illnesses: past, present, and future. Epidemiol Camb Mass. 2005;16(6):791-801.

5. Li Q, Guan X, Wu P, Wang X, Zhou L, Tong Y, et al. Early Transmission Dynamics in Wuhan, China, of Novel Coronavirus-Infected Pneumonia. N Engl J Med. Accessed on 23 March 2020.

6. CDC. Coronavirus Disease 2019 (COVID-19). Centers for Disease Control and Prevention. 2020. Available from: https://www.cdc.gov/coronavirus /2019-ncov/hcp/therapeutic-options.html. Accessed on 23 March 2020.

7. Prajapat M, Sarma P, Shekhar N, Avti P, Sinha S, Kaur $\mathrm{H}$, et al. Drug targets for corona virus: A systematic review. Ind J Pharmacol. 2020;52(1):56.

8. Lotteau V, Teyton L, Peleraux A, Nilsson T, Karlsson L, Schmid SL, et al. Intracellular transport of class II MHC molecules directed by invariant chain. Natu. 1990;348(6302):600-5.

9. Wu SF, Chang CB, Hsu JM, Lu MC, Lai NS, Li C, et al. Hydroxychloroquine inhibits CD154 expression in CD4+ $\mathrm{T}$ lymphocytes of systemic lupus erythematosus through NFAT, but not STAT5, signalling. Arthritis research \& therapy. 2017;19(1):183.

10. Dijkmans BA, Verweij CL. Chloroquine and hydroxychloroquine equally affect tumor necrosis factor-alpha, interleukin 6, and interferon-gamma production by peripheral blood mononuclear cells. J Rheumatol. 1997;24(1):55-60.

11. Kužnik A, Benčina M, Švajger U, Jeras M, Rozman B, Jerala R. Mechanism of endosomal TLR inhibition by antimalarial drugs and imidazoquinolines. J Immunol. 2011;186(8):4794-804.

12. An J, Woodward JJ, Sasaki T, Minie M, Elkon KB. Cutting edge: antimalarial drugs inhibit IFN- $\beta$ production through blockade of cyclic GMP-AMP synthase-DNA interaction. J Immunol. 2015;194(9):4089-93.

13. Caly L, Druce JD, Catton MG, Jans DA, Wagstaff KM. The FDA-approved Drug Ivermectin inhibits the replication of SARS-CoV-2 in vitro. Antivir Resea. 2020:104787.

14. Gielen V, Johnston SL, Edwards MR. Azithromycin induces anti-viral responses in bronchial epithelial cells. Europe Respirat J. 2010;36(3):646-54.

15. Shinkai M, Henke MO, Rubin BK. Macrolide antibiotics as immunomodulatory medications: proposed mechanisms of action. Pharmacol Therapeut. 2008;117(3):393-405.

16. Gautret P, Lagier JC, Parola P, Meddeb L, Mailhe M, Doudier B, et al. Hydroxychloroquine and azithromycin as a treatment of COVID-19: results of an open-label non-randomized clinical trial. Int J Antimicrob Agen. 2020:105949.
17. Ventricular Arrhythmia Risk Due to Hydroxychloroquine-Azithromycin Treatment For COVID-19 - American College of Cardiology. Available at: https://www.acc.org/latest-in-cardiolo gy/articles/2020/03/27/14/00/ventricular-arrhythmiarisk-due-to-hydroxychloroquine-azithromycintreatment-for-covid-19. Accessed on 16 April 2020.

18. Xue X, Yu H, Yang H, Xue F, Wu Z, Shen W, et al. Structures of two coronavirus main proteases: implications for substrate binding and antiviral drug design. J Virol. 2008;82(5):2515-27.

19. Cao B, Wang Y, Wen D, Liu W, Wang J, Fan G, et al. A trial of lopinavir-ritonavir in adults hospitalized with severe Covid-19. N Engla J Medic. 2020;18.

20. A Multi-centre, Double-blinded, Randomized, Placebo-controlled Trial on the Efficacy and Safety of Lopinavir/Ritonavir Plus Ribavirin in the Treatment of Severe Acute Respiratory SyndromeClinicalTrials.gov. Available at: https://clinicaltrials. gov/ct2/show/NCT00578825. Accessed on 16 April 2020.

21. Welliver R, Monto AS, Carewicz O, Schatteman E, Hassman M, Hedrick J, et al. Oseltamivir Post Exposure Prophylaxis Investigator Group. Effectiveness of oseltamivir in preventing influenza in household contacts: a randomized controlled trial. J Americ Medic Assoc. 2001;285(6):748-54.

22. Agostini ML, Andres EL, Sims AC, Graham RL, Sheahan TP, Lu X, et al. Coronavirus susceptibility to the antiviral remdesivir (GS-5734) is mediated by the viral polymerase and the proofreading exoribonuclease. MBio. 2018;9(2):e00221-18.

23. A Trial of Remdesivir in Adults with Mild and Moderate COVID-19- ClinicalTrials.gov. Available at: https://clinicaltrials.gov/ct2/show/NCT04252664. Accessed on 16 April 2020.

24. Furuta Y, Takahashi K, Kuno-Maekawa M, Sangawa H, Uehara S, Kozaki K, et al. Mechanism of action of T-705 against influenza virus. Antimicrob Agen Chemother. 2005;49(3):981-6.

25. Lu R, Zhao X, Li J, Niu P, Yang B, Wu H, et al. Genomic characterisation and epidemiology of 2019 novel coronavirus: implications for virus origins and receptor binding. Lanc. 2020;395(10224):565-74.

26. Elfiky AA. Anti-HCV, nucleotide inhibitors, repurposing against COVID-19. Life scienc. 2020:117477.

27. Xu X, Han M, Li T, Sun W, Wang D, Fu B, et al. Effective Treatment of Severe COVID-19 Patients with Tocilizumab. Chin Xiv prepr. 2020.

28. Cohort Multiple Randomized Controlled Trials Open-label of Immune Modulatory Drugs and Other Treatments in COVID-19 Patients - Sarilumab Trial CORIMUNO-19-SARI-ClinicalTrials.gov. Available at: https://clinicaltrials.gov/ct2/show/NCT04324073. Accessed on 16 April 2020.

29. Sorrell FJ, Szklarz M, Azeez KR, Elkins JM, Knapp $\mathrm{S}$. Family-wide structural analysis of human numbassociated protein kinases. Struct. 2016;24(3):401-11. 
30. Sallard E, Lescure FX, Yazdanpanah Y, Mentre F, Peiffer-Smadja N, Florence AD, et al. Type 1 interferons as a potential treatment against COVID19. Antivir Resea. 2020:104791.

31. Trial of Treatments for COVID-19 in Hospitalized Adult. Available at: https://clinicaltrials.gov/ct2/ show/NCT04315948. Accessed on 16 April 2020.

32. Biocon Limited. A Multi-Centre, Open Label, Two Arm Randomized, Pivotal Phase 2 Trial to Study the Efficacy and Safety of Itolizumab in COVID-19 Complications. Available at: https://clinicaltrials.gov /ct2/show/NCT04475588. Accessed on 02 July 2020.

33. Biocon's Itolizumab Approved in India for COVID19 Inflammation. Available at: https://www.center forbiosimilars.com/view/biocons-itolizumab-approv ed-in-india-for-covid19-inflammation. Accessed on 05 July 2020.

34. Srivastava A. Itolizumab in Psoriasis. Indian $\mathbf{J}$ Dermatol. 2017;62(4):418-21.

35. Loganathan S, Athalye SN, Joshi SR. Itolizumab, an anti-CD6 monoclonal antibody, as a potential treatment for COVID-19 complications. Expert Opin Biol Ther. 2020;1-7.

36. Chinese Clinical Trial Register (ChiCTR) - The world health organization international clinical trials registered organization registered platform. Available at: http://www.chictr.org.cn/abouten.aspx. Accessed on16 April 2020.

37. Safety and Immunogenicity Study of 2019-nCoV Vaccine (mRNA-1273) for Prophylaxis SARS CoV-2 Infection (COVID-19). Available at: https://clinical trials.gov/ct2/show/NCT04283461. Accessed on 16 April 2020.

38. Reducing Health Care Workers Absenteeism in Covid-19 Pandemic Through BCG Vaccine. Available at: https://clinicaltrials.gov/ct2/show/NCT 04328441. Accessed on 16 April 2020.

39. COVAXIN. India's First Indigenous Covid-19 Vaccine. Bharat Biotech. Available at: https://www.bharatbiotech.com/covaxin.html. Accessed on 7 August 2020.

40. Bharat Biotech International Ltd. An Adaptive, Seamless Phase 1, Followed by Phase 2 Randomized, Double-blind, Multicentre Study to Evaluate the Safety, Reactogenicity, Tolerability and Immunogenicity of the Whole-Virion Inactivated SARS-CoV-2 Vaccine (BBV152) in Healthy Volunteers. Available at: http://ctri.nic.in/Clinical trials/showallp.php? $\operatorname{mid} 1=45184 \&$ EncHid $=\&$ userNa me=bbv152. Last accessed on 10 August 2020.

41. Kirby T. Development of potential COVID-19 vaccines continues to accelerate. Lancet Microbe. 2020;1(3):e109.

42. Chugh T. Timelines of COVID-19 Vaccines. Curr Med Res Pract. 2020

43. Shen C, Wang Z, Zhao F, Yang Y, Li J, Yuan J, et al. Treatment of 5 critically ill patients with COVID-19 with convalescent plasma. J Americ Medic Assoc. 2020 .
44. Hattori A, Migitaka H, Iigo M, Itoh M, Yamamoto K, Ohtani-Kaneko R, et al. Identification of melatonin in plants and its effects on plasma melatonin levels and binding to melatonin receptors in vertebrates. Biochem Mol Biol Int. 1995;35(3):627-34.

45. Boga JA, Coto-Montes A, Rosales-Corral SA, Tan D-X, Reiter RJ. Beneficial actions of melatonin in the management of viral infections: a new use for this "molecular handyman"? Rev Med Virol. 2012;22(5):323-38.

46. Results- RECOVERY Trial. Available at: https://www.recoverytrial.net/results. Accessed on 20 June 2020.

47. Fang L, Karakiulakis G, Roth M. Are patients with hypertension and diabetes mellitus at increased risk for COVID-19 infection? The Lanc. Respirat Medic.

48. Pal R, Bhansali A. COVID-19, Diabetes Mellitus and ACE2: The conundrum. Diabet Resear Clinic Practi. 2020.

49. Wang M, Cao R, Zhang L, Yang X, Liu J, Xu M, et al. Remdesivir and chloroquine effectively inhibit the recently emerged novel coronavirus (2019-nCoV) in vitro. Cell Resea. 2020;30(3):269-71.

50. Yao X, Ye F, Zhang M, Cui C, Huang B, Niu P, et al. In vitro antiviral activity and projection of optimized dosing design of hydroxychloroquine for the treatment of severe acute respiratory syndrome coronavirus 2 (SARS-CoV-2). Clinic Infect Diseas. 2020.

51. Geleris J, Sun Y, Platt J, Zucker J, Baldwin M, Hripcsak G, et al. Observational study of hydroxychloroquine in hospitalized patients with Covid-19. N Engla J Medic. 2020.

52. Beigel JH, Tomashek KM, Dodd LE, et al. Remdesivir for the Treatment of Covid-19Preliminary Report. N Engl J Med. 2020;10.

53. Chu CM, Cheng VC, Hung IF, Wong MM, Chan $\mathrm{KH}$, Chan KS, et al. Role of lopinavir/ritonavir in the treatment of SARS: initial virological and clinical findings. Thor. 2004;59(3):252-6.

54. Cao B, Wang Y, Wen D, Liu W, Wang J, Fan G, et al. A trial of lopinavir-ritonavir in adults hospitalized with severe Covid-19. N Engla J Medic. 2020.

55. A Randomized, Open, Controlled Clinical Study to Evaluate the Efficacy of ASC09F and Ritonavir for 2019-nCoV Pneumonia. Available at: https://clini caltrials.gov/ct2/show/NCT04261270. Accessed on 16 April 2020.

56. WHO. Blueprint. Priority Disease. Available at: https://www.who.int/blueprint/priority-diseases/key action/Novel_Coronavirus_Landscape_nCoV_PDF. Accessed on 16 April 2020.

57. FDA. Research $\mathrm{C}$ for DE and. Coronavirus Treatment Acceleration Program (CTAP). FDA. Available at: https://www.fda.gov/drugs/coronavirus-covid-19drugs/coronavirus-treatment-acceleration-programctap. Accessed on 16 April 2020.

58. Silvestri M, Rossi GA. Melatonin: its possible role in the management of viral infections-a brief review. Itali J Paediat. 2013;39(1):61. 
59. Zhou P, Yang XL, Wang XG, Hu B, Zhang L, Zhang $\mathrm{W}$, et al. A pneumonia outbreak associated with a new coronavirus of probable bat origin. Natu. 2020:1-4.

60. Gitto E, Reiter RJ, Amodio A, Romeo C, Cuzzocrea E, Sabatino G, et al. Early indicators of chronic lung disease in preterm infants with respiratory distress syndrome and their inhibition by melatonin. J Pine Resea. 2004;36(4):250-5.

61. World Health Organization. WHO welcomes preliminary results about dexamethasone use in treating critically ill COVID-19 patients. Available at: https://www.who.int/news-room/detail/16-062020-who-welcomes-preliminary-results-about- dexamethasone-use-in-treating-critically-ill-covid19-patients. Accessed on 18 June 2020 June.

62. World Health Organization. Clinical management of severe acute respiratory infection when novel coronavirus ( $\mathrm{nCoV}$ ) infection is suspected. Interim guidance. 2020.

Cite this article as: Kumar V, Vardhan G, Tiwari K, Dhamija P. A review of potential pharmacological treatments of COVID-19: an evidence-based perspec tive. Int J Basic Clin Pharmacol 2020;9:1757-65. 